\section{Ulzerationen an den Füßen und schuppende Erytheme an Fingerrücken und Zehen}

\section{Anamnese}

Ein 65-jähriger Mann stellte sich wegen seit einem Jahr progredienter, therapieresistenter Ulzerationen an Füßen, Fußsohlen und Knöchelregionen vor. Weiterhin bemerkte er seit 4 Jahren an den Hand- und Fußrücken zunehmende Rötungen mit Schuppung, welche zeitweise jucken würden.

Vorerkrankungen: Seit 18 Jahren bekannte chronisch-myeloische Leukämie. Zunächst über 6 Jahre Therapie mit Busulfan $\left(\right.$ Myleran $\left.^{\circledR}\right)$, anschließend seit 12 Jahren systemische Gabe von Hydroxyurea (Litalir ${ }^{\circledast}$ ), zuletzt $3000 \mathrm{mg}$ täglich. Zwischenzeitlich über 6 Jahre zusätzliche Therapie mit Interferon alpha (Intron $A^{\circledR}$ ), die bei Verdacht auf vaskulitische Veränderungen an den Händen abgesetzt wurde. Seit 6 Jahren insulinpflichtiger Diabetes mellitus Typ IIb und arterielle Hypertonie.

Aktuelle Medikation: Hydroxyurea $3000 \mathrm{mg} / \mathrm{die}$, Ramipril $10 \mathrm{mg} /$ die, Triamteren $50 \mathrm{mg} / \mathrm{die}$, Hydrochlorothiazid $25 \mathrm{mg} /$ die, humanes Depot-Insulin 20-0-8 IE/die.

\section{Dermatologischer Befund}

An Hand- und Fingerrücken mit Betonung der Metakarpophalangeal- und Interphalangealgelenke streifige, schuppende Erytheme mit atrophischen, z. T. hyperpigmentierten, z. T. lividen Arealen (Abb.1). Gleichartige Hautveränderungen in geringerer Ausprägung an den Zehenrücken mit Übergreifen auf die Fußrücken und Fußsohlen.

An den Fußsohlen, der rechten Großzehe und der 2. Zehe rechts 4 sehr schmerzhafte, maximal $11 \times 8 \mathrm{~mm}$ große Hyperkeratosen mit zentraler Ulzeration; am rechten Außenknöchel ein $30 \times 16 \mathrm{~mm}$ großes, schmierig belegtes Ulkus (Abb. 2).
B. Schubert, U. Wesselmann, Eva-B. Bröcker, H. Hamm

Universitäts-Hautklinik Würzburg

(Direktorin: Prof. Dr. med. Eva-B. Bröcker)

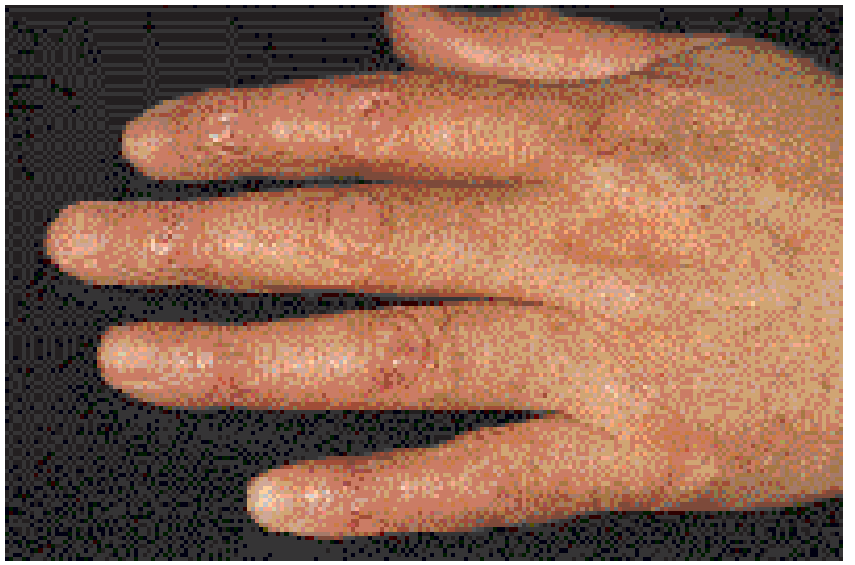

Abb. 1 Klinischer Befund der linken Hand.

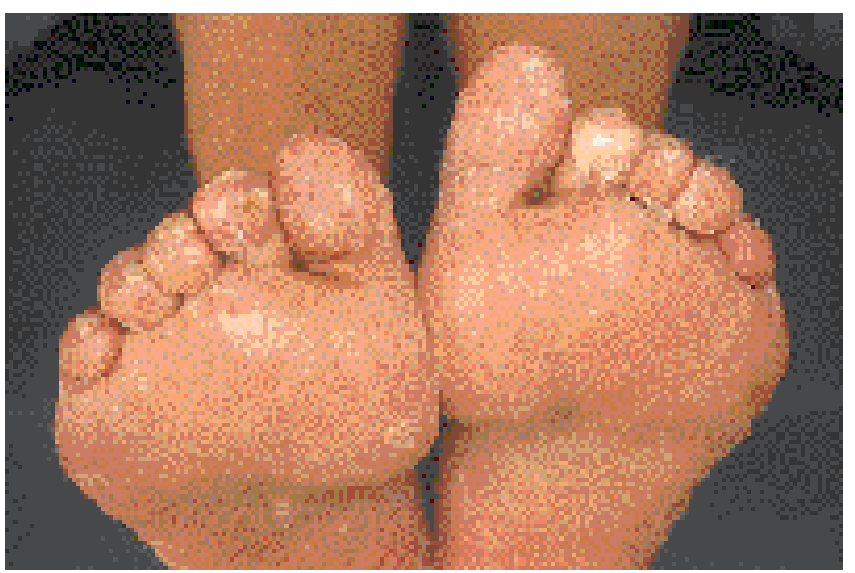

Abb. 2 Klinischer Befund der Vorfüße. 


\section{Histopathologie}

Probebiopsie (rechter Handrücken): Kompakte massive Hyperkeratose mit z.T. parakeratotischer Verhornung. Diskrete Akanthose, fokal geringe Atrophie der Epidermis, leichte Zellund Kernpolymorphie der basalen Keratinozyten. Zahlreiche erweiterte Blutgefäße sowie diskrete Fibrosierung in der oberen Dermis, kein Entzündungsinfiltrat (Abb. 3).

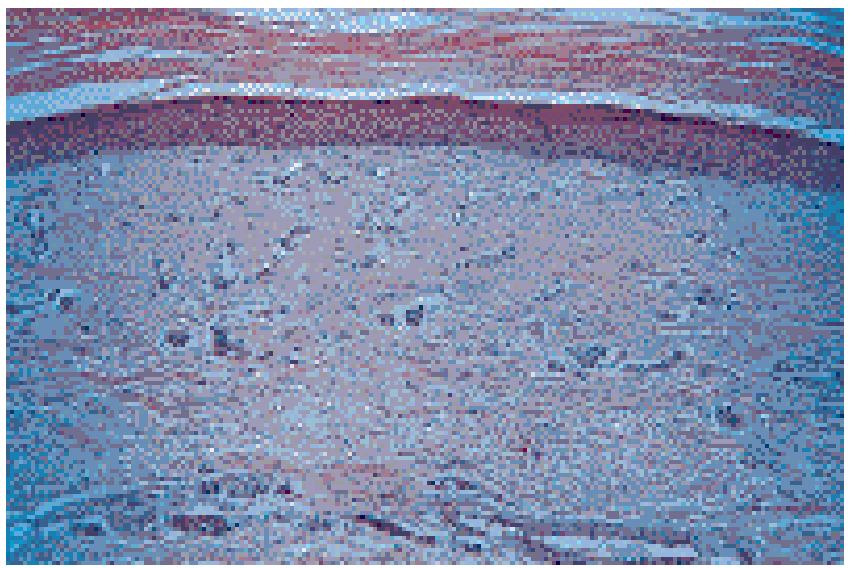

Abb. 3 Histologischer Befund einer Biopsie vom rechten Handrücken $(\mathrm{HE}, \times 400)$.

\section{Labor und Zusatzuntersuchungen}

BSG 31/65 mm n.W., Kalium 5,5 mmol/l, Glukose $104 \mathrm{mg} / \mathrm{dl}$,

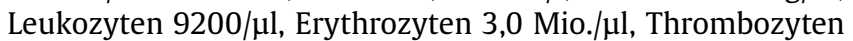

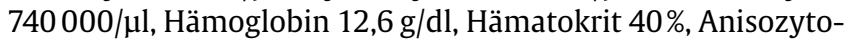
se, Makrozytose, übriges Routinelabor unauffällig. Kreatinkinase im Serum normwertig. Kreatin im 24-h-Urin und Kreatinin-Clearance normwertig. ANA-Titer 1:160, homogenes Muster ohne Mitosen. ENA-Screen negativ. Gesamt-IgE auf $487 \mathrm{kU} /$ 1 erhöht. AST auf 4,0 IE/ml erhöht, ASL normwertig, CRP auf $12,7 \mathrm{mg} / \mathrm{l}$ erhöht. Borrelien-Serologie: IgG im ELISA und Western-Blot positiv, im Immunfluoreszenztest (IFT) grenzwertig (Titer 1:20), IgM im ELISA und IFT negativ.

Mykologie: Füße und Zehennägel: Trichophyton rubrum. Hände und Fingernägel: negativ.

Bakteriologie: Ulzera: massenhaft Staphylococcus aureus.

Neurologische Untersuchung: Periphere sensorische Polyneuropathie.

Phlebologische Untersuchung: Immobilisationsbedingtes Stauungssyndrom ohne Nachweis einer chronisch-venösen Insuffizienz des tiefen und oberflächlichen Venensystems.

Farbkodierte Duplexsonographie der Becken-Beinarterien: Zeichen einer Mediasklerose, distale Okklusion der rechten Arteria tibialis posterior ohne hämodynamische Relevanz.

Auflösung auf S. 243 
Auflösung von S. 242

\section{Diagnose: Fußulzerationen und dermatomyositis- artige Hautveränderungen unter Langzeittherapie mit Hydroxyurea (Litalir ${ }^{\circledR}$ )}

\section{Kommentar}

Bei den dermatomyositisartigen Hautveränderungen an Händen und Füßen handelt es sich um eine relativ seltene, aber substanzspezifische Nebenwirkung des Chemotherapeutikums Hydroxyurea (Litalir ${ }^{\circledR}$ ). Hydroxyurea findet seine Anwendung bei hämatologischen Erkrankungen, wie der chronisch-myeloischen Leukämie, der Polycythaemia vera und der essenziellen Thrombozythämie. Es ist ein Antimetabolit und inhibiert durch Hemmung der Ribonukleotid-Reduktase die DNA-Synthese $[1,2,8]$.

Die dermatomyositisartigen Hautveränderungen unter Hydroxyurea sind durch streifige, schuppende Erytheme mit poikilodermatischem Aspekt gekennzeichnet $[1,2,9,10,12]$. Typische Lokalisationen sind die Hand- und Fingerrücken mit Betonung der Metakarpophalangeal- und Interphalangealgelenke. Seltener sind auch die Fußrücken und Zehen betroffen. Differenzialdiagnostisch muss an eine Dermatomyositis gedacht werden. Klinisch fehlen allerdings Muskelschmerzen oder-schwäche; elektromyographische Veränderungen lassen sich nicht nachweisen. Die lividen periorbitalen Erytheme und Ödeme einer klassischen Dermatomyositis sind üblicherweise ebenfalls nicht vorhanden. Allerdings sind auch Fälle mit periorbitalen Ödemen und Gesichterythemen unter Hydroxyurea beobachtet worden [1]. Laborchemisch ist die Kreatinkinase normwertig, auch das Kreatin im 24-Stunden-Urin ist nicht erhöht.

Histologisch werden eine Orthohyperkeratose, epidermale Atrophie, eine Interface-Dermatitis mit schütterem lichenoiden Infiltrat und Teleangiektasien in der oberen Dermis beschrieben $[1,2,9,10]$. In der von uns gewonnenen Biopsie fand sich allerdings kein nennenswertes Entzündungsinfiltrat.

Typischerweise treten die Hautveränderungen nach einer Behandlungsdauer von 16-35 Monaten auf und sind nach Absetzen von Hydroxyurea reversibel. Da sie subjektiv jedoch meist nicht sehr beeinträchtigend sind und eine dringende Therapienotwendigkeit der hämatologischen Grunderkrankung besteht, ist es in der Regel nicht indiziert, die Medikation abzusetzen. In unserem Falle erreichten wir eine Besserung durch eine Lokaltherapie mit Dexpanthenol-Salbe unter Okklusion und nachfolgender Hautpflege mit einem harnstoffhaltigen Präparat.

Die Pathogenese der dermatomyositisartigen Hautveränderungen unter Langzeittherapie mit Hydroxyurea ist unklar. Da Hydroxyurea die DNA-Synthese beeinträchtigt, wird angenommen, dass auch die Zellteilung der basalen Keratinozyten gehemmt wird. UV-Licht verstärkt möglicherweise die Wirkung, wofür die bevorzugte Lokalisation an den Hand- und Fingerrücken spricht [1].
Weitere, häufige Nebenwirkungen von Hydroxyurea sind Ulzerationen [11 - 17]. Kennzeichnend sind die bevorzugte Lokalisation an den lateralen bzw. medialen Malleoli oder in der Perimalleolarregion, die häufig rundliche Form und die ausgeprägte Schmerzhaftigkeit. Die Ulzerationen treten nach 1 - bis 10-jähriger Hydroxyurea-Therapie bei einer täglichen Dosis von mindestens $1000 \mathrm{mg}$ auf. Eine Korrelation zwischen der Dosis und der Ausprägung der Ulzerationen wird angenommen. Die Ulzera entstehen spontan oder nach Mikrotraumen und treten oft gemeinsam mit dermatomyositisartigen Hautveränderungen an Händen und Füßen auf. Die Ulzerationen sind außerordentlich therapierefraktär, zeigen jedoch nach Absetzen der Medikation eine rasche Heilungstendenz.

Pathogenetisch wird ein direkter Effekt von Hydroxyurea auf die Ulkusentstehung angenommen. Hydroxyurea hemmt die DNA-Synthese und somit auch die Proliferation von Endothelien, Fibroblasten und Keratinozyten. Hautatrophie und Mikrotraumen führen im Verbund mit der verminderten Wundheilung zur Ulzeration. Die durch die Thrombozytose bedingte verminderte Mikrozirkulation hat sicherlich ebenfalls einen Anteil an der Ulkusentstehung. Zusätzliche arterielle oder venöse Dysfunktionen verschlechtern die Heilung.

Auch in unserem Falle ist die Pathogenese der Ulzerationen nicht ganz eindeutig. Sicherlich trugen der Diabetes mellitus und möglicherweise auch die arterielle Gefäßsituation zur Entstehung der Ulzera bei. Allerdings sprechen der zeitliche Zusammenhang, die typische Lokalisation, die Multiplizität und die Schmerzhaftigkeit der Ulzera sowie ihre geringe Heilungstendenz für eine wesentliche Beteiligung des Hydroxyurea an der Ulkusgenese.

Weitere Nebenwirkungen einer Hydroxyurea-Langzeittherapie treten in etwa 10-35\% auf (siehe Tab. 1). Die meisten dieser Veränderungen sind relativ unspezifisch und werden auch bei anderen Zytostatika beobachtet [1-8, 11].

Tab.1 Nebenwirkungen einer Hydroxyurea-Langzeittherapie

- Ulzerationen

- dermatomyositisartige Hautveränderungen

- Xerosis cutis

- Hyperpigmentierung bevorzugt an druckexponierten Stellen

- Plantarkeratosen

- fixe Arzneimittelexantheme

- Gesichts-, Hand- und Fingererytheme

- Begünstigung von aktinischen Keratosen, kutanen Stachelzellund Basalzellkarzinomen

- Stomatitis mit Ulzerationen

- Effluvium

- Alopezie

- Nagelveränderungen: Onycholysis, Onychodystrophie und charakteristische longitudinale Melanonychie 


\section{Literatur}

${ }^{1}$ Weber L, Schick E, Merkel M, Pillekamp H, Sterry W. Dermatomyositisartige Hautveränderungen unter Hydroxyurea (Litalir)Langzeittherapie. Hautarzt 1995; 46: 717-721

${ }^{2}$ Grange F, Couilliet D, Audhuy B, Krzisch S, Schlecht P, Guillaume JC. Kératoses multiples induites par l'hydroxyurée. Ann Dermatol Venereol 1995; 122: 16 - 18

${ }^{3}$ Gropper CA, Don PC, Sadjadi MM. Nail and skin hyperpigmentation associated with hydroxyurea therapy for polycythemia vera. Int J Dermatol 1993; 32: $731-733$

${ }^{4}$ Vomvouras S, Pakula AS, Shaw JM. Multiple pigmented nail bands during hydroxyurea therapy: an uncommon finding. J Am Acad Dermatol 1991; 24: 1016 - 1017

${ }^{5}$ Angeli-Besson C, Koeppel MC, Jacquet P, Andrac L, Sayag J. Multiple squamous-cell carcinomas of the scalp and chronic myeloid leukemia. Dermatology 1995; 191: 321 - 322

${ }^{6}$ Salmon-Ehr V, Grosieux C, Potron G, Kalis B. Multiple actinic keratosis and skin tumors secondary by hydroxyurea treatment. Dermatology 1998; 196: 274

${ }^{7}$ De Simone C, Guerriero C, Guidi B, Rotoli M, Venier A, Tartaglione F. Multiple squamous cell carcinomas of the skin during longterm treatment with hydroxyurea. Eur J Dermatol 1998; 8: 114 115

${ }^{8}$ Sigal M, Crickx B, Blanchet P, Perron J, Simony J, Belaich S. Lésions cutanées induites par l'utilisation au long cours de l'hydroxyurée. Ann Dermatol Venereol 1984; 111: 895-900

${ }^{9}$ Daoud MS, Gibson LE, Pittelkow MR. Hydroxyurea dermopathy: a unique lichenoid eruption complicating long-term therapy with hydroxyurea. J Am Acad Dermatol 1997; 36: 178 - 182

${ }^{10}$ Richard M, Truchetet F, Friedel J, Leclech C, Heid E. Skin lesions simulating chronic dermatomyositis during long-term hydroxyurea therapy. J Am Acad Dermatol 1989; 21: 797-799

${ }^{11}$ Ravandi-Kashani F, Cortes J, Cohen P, Talpaz M, O'Brian S, Markowitz A, Kantarjian H. Cutaneous ulcers associated with hydroxyurea therapy in myeloproliferative disorders. Leuk Lymphoma 1999; 35: 109-118

12 Varma S, Lanigan SW. Dermatomyositis-like eruption and leg ulceration caused by hydroxyurea in a patient with psoriasis. Clin Exp Dermatol 1999; 24: $164-166$

${ }^{13}$ Weinlich G, Schuler G, Greil R, Kofler H, Fritsch P. Leg ulcers associated with long-term hydroxyurea therapy. J Am Acad Dermatol 1998; 39: $372-374$

${ }^{14}$ Suehiro M, Kishimoto S, Wakabayashi T, Ikeuchi A, Miyake H, Takenaka H, Okano A, Hirai H, Shimazaki C, Yasuno H. Hydroxyurea dermopathy with a dermatomyositis-like eruption and a large leg ulcer. Br J Dermatol 1998; 139: 748 - 749

${ }^{15}$ Weinlich G, Fritsch P. Leg ulcers in patients treated with hydroxyurea for myeloproliferative disorders: what is the trigger? $\mathrm{Br} \mathrm{J}$ Dermatol 1999; 141: $171-172$

${ }^{16}$ Bader U, Banyai M, Böni R, Burg G, Hafner J. Leg ulcers in patients with myeloproliferative disorders: disease- or treatment-related? Dermatology 2000; 200: 45 - 48

17 Sirieix ME, Debure C, Baudot N, Dubertret L, Roux ME, Morel P, Frances C, Loubeyres S, Beylot C, Lambert D, Humbert P, Gauthier O, Dandurand M, Guillot B, Vaillant L, Lorette G, Bonnetblanc JM, Lok C, Denoeux JP. Leg ulcers and hydroxyurea. Arch Dermatol 1999; 135: $818-820$

\section{B. Schubert}

Universitäts-Hautklinik

Josef-Schneider-Straße 2

97080 Würzburg

E-mail: Schubert-b.derma@mail.uni-wuerzburg.de 